\title{
Taking students outside the classrooms. Location-based mobile games in education
}

\author{
Mauro Ceconello, Davide Spallazzo, Martina Sciannamè
}

Department of Design, Politecnico di Milano, Italy.

\begin{abstract}
The contribution aims at corroborating location-based mobile games as models for the integration of digital technologies in the educational field. They demonstrated to be valid alternatives to formal education in the applied research project: Play Design!, which addressed to high school students, interested in design-related matters, and intends to valorise the Italian design culture, transforming Milan into the stage of a double-sided story. Design is here highlighted both as a cultural heritage and a discipline, inducing the development of two different games sharing a common didactic aim: D.Hunt and D.Learn. The first one is a mobile treasure hunt illustrating the excellences of the creative production of the country, and the renowned protagonists and places of Italy- and Milan-based design: a cultural background to be preserved and valorised. The second one, instead, is a roleplay, cooperative and competitive game which depicts the city as a hub for schools and universities, where design is considered a subject for didactic courses, a combination of theories and practices to be transmitted and implemented. Then, the two mobile, location-based serious games exploit this copious and multifaceted material for evident learning purposes, joining the examples of informal education to increasingly follow in future technology developments.
\end{abstract}

Keywords: Location-based mobile games; informal learning; serious games; technology in education; situated experience. 


\section{LBMGs as models for integrating digital technology in education}

Digital technologies have been spreading more and more pervasively in our current society, at the extent that, since a few years, they penetrated in schools with MOOCs (Massive Open Online Courses), IWBs (Interactive Whiteboards), several educational apps and, currently, AI is gaining ground with software like Gradescope or IBM Watson Education. Yet, in the field of education, since 1980's, AI has been mostly investigated in a knowledge-based approach (Tuomi, 2018, p. 27), and students considered little pitchers to be filled with facts, in Dickens's words (1854, p. 5). According to some studies, machines can even substitute teachers in their mundane tasks: revising, grading and even providing notions for students to learn, in addition to supporting them as ITSs (Intelligent Tutoring Systems), and reaching a universal range of different users (Nafea, 2018; Tuomi, 2018). What emerges, though, is a step backwards from a UX point of view and computers seem to have come back to a private relationship with humans, isolating them as it happened in the early era of videogames consoles. Probably, the application of AI systems to education should take in consideration the development of serious digital games as a model. Whether they are a consolidated but not widely diffused approach in education, location-based mobile games (LBMGs henceforth), for instance, go beyond the behaviourist and cognitivist generations of games, laying in pedagogical, experiential and situated learning paradigms (Ulicsak \& Wright, 2010). Then, the emphasis is moved from factual education to the improvement of soft skills, which are much needed in a society where automation may overcome many working positions. Collaborative, social and situated dimensions become pivotal in LBMGs, allowing meaning-making through cooperation, coordination and cocreation (McGonigal, 2011), but also in relation to their physical context. This also responds to a contemporary emergency: young students are constantly influenced by rapid digital information and social connections. The challenge is to motivate them to actively participate in an educational experience that fulfils their expectations, proposing them something compelling an enjoyable, fun and cool (Anastasiadis, Lampropoulo, \& Siaka, 2018; Read, 2015). To this end, mobile technology has already proved its efficacy, being the protagonist of a new common cultural practice (Mariani, 2014), that brought games and, then, learning in real-world contexts, matching interactive engagement with serious learning (Prensky, 2001), in contact with authentic environments (Huizenga, Admiraal, Akkerman, \& Dam, 2009). Digital game-based learning fosters not only game-related skills, but players get acknowledged about specific topics - according to the subject of the game - (Gee, 2003, 2004), and may be influenced in their real life behaviour through active engagement and situated learning (Lave \& Wenger, 1991). Taking learning out of classrooms and formal education, thanks to informal and playful activities, thus demonstrates the effectivity of situatedness in physical spaces, where already present and digitally overlaid contents hybridize in a more meaningful world (Klopfer, 2008; Klopfer, 
Squire, \& Jenkins, 2002), and where social, authentic, open-ended, intrinsically motivating and filled with feedback educating experiences (Klopfer, 2008) may take place. Yet not technology, but its purposes and the entire system in which it is employed are relevant in fostering learning, especially when related to a project of cultural heritage.

In the following dissertation, a case study is presented as an example of LBMGs supporting educational purposes through a wise calibration of informal, playful and engaging experiences.

\section{The design of LBMGs as educational tools}

To valorise the design culture of Milan, in the Play Design! project, the city acquires new values as a rich playground, full of meaningful contents that, at the same time, highlight the glorious traces of past design interventions and the current approaches of design schools and universities. Though, in spite of this duality of possible communication - resulting into two different mobile games - the target users are common to both the experiences. In fact, they have been developed to attract and inform high school students interested in the field of design. This is the reason why the Politecnico di Milano Open Day, where the school presented its undergraduate offers to students perfectly matching the target, has been detected as a positive occasion for the promotion of the project, D.Hunt in particular. While, the launch of D.Learn required precise conditions and necessitated a further step of registration and consent, therefore bringing to a direct contact with local high schools. Then a specific event followed, as it will be explicated later on.

To better engage the young public and properly communicate the unique identity of the project, both games share a common contemporary graphic language, which is based on bright colour variations and a simplified geometric style. Also the logo is playful and captivating: it is inspired by Munari's Zizì Monkey (protagonist of D.Hunt), which is differently portrayed for the two games, and in the promotional tools (postcards, pins, website, digital fabricated rewarding medals, etc.). The customisation of the games' user interface, instead, is limited, because the development of the mobile learning experiences has been realized with the support of a bespoke commercial platform. This choice has been due to limited resources and to the aim of the project, that was not focused on the generation of a new app but on the testing of LBMGs as educating tools capable of communicating the design traces across the city. Additionally, it allowed an optimisation of the iterative process employed to verify the functionality of the games. Before the launch, they were tested several times: firstly, by the research team, and subsequently involving a sample of final users. Actually, most of the test and correction phase was focused on the contents and on the user experience, because basic development problems were avoided 
using the off-the-shelf platform, limiting the errors to simple bugs and mistakes in the location of the Points of Interest (PoIs henceforth) and typos.

Apart from the above-mentioned points of contact, related to a coordinated organization of the project, this had to respond to different purposes. For that reason, narrative, game tools and mechanics, and, consequently, the methods of assessment of the experiences were differentiated.

\subsection{D.Hunt}

Targeting high-school students who are curious about design and who want to enjoy an engaging activity, D.Hunt aims at fostering the recognition of design traces in Milan. Here, renowned industrial products, designers and brands, iconically representing the Italian design in the world, are intended as a precious cultural heritage to be discovered. That is why the game genre of treasure hunt seemed to be the most effective approach, on which to build the narrative providing information and contextualization to the subject.

To encourage involvement and active participation in a single player experience, the story begins with a mystery: the famous Zizì monkey has been kidnapped and the player is the detective designer appointed to find it, following its traces. Zizì monkey fulfils the double task of narrative device and Cicero, guiding the players through the streets of Milan to make them discover institutions, showrooms, and important public places of the history of Italian and Milanese design. The pet toy is actually the virtual protagonist of the treasure hunt and it highly marks the narrative register. In fact, the monkey directly interacts with the player asking questions and giving clues, not only through the visual interface of the app, but additionally by means of audio tracks that simulate a radio broadcast providing design-related notions. During the speaker's narration, in fact, Zizì intrudes in the transmission to suggest the next move. This tool contributes, once again, to link the different locations in terms of plot and physical path, to foster learning by a curious and informal channel, and to lighten the entire experience, making it more enjoyable. All of this results in a friendly and informal tone throughout the entire game, from the description of the rules, to the narrative and the questions.

The game develops completely in the intertwinement of the digital mobile experience and physical relevant spaces. It starts at the Bruno Munari Association (accordingly to the protagonist of the story) as the first of fourteen locations in downtown Milan. There, thanks to the GPS localization of the player's smartphone, the app provides the first hint, opening a path of investigation through clues and questions regarding the history of Italian design, icons (products, interiors or graphics) or designers strongly related to the places where they are triggered. So, the player has to figure out the correct answers to proceed in his mission and reach the following location, activating a process of informal learning. Each question has multiple answers and one single error is allowed, causing, as a result, the appearance of 
an additional hint. The difficulty level of the game is medium-low to meet the knowledge of a young audience which is only interested in design related matters but not strictly specialized: detailed descriptions or images are provided, facilitating the player's correct inferring. When the player manages to solve the enigma, at the end of the treasure hunt, each solved task is assigned with a score based on the number of correct answers provided and the performance is accordingly evaluated as: a Sherlock of investigations, a good detective, a promising apprentice or an inexperienced beginner.

Regarding the assessment of the experience, in this case, it was difficult to have significant and first-hand feedbacks, as the game can be easily played autonomously and without supervision. The only meter of evaluation derived from an up to five stars rating and simple digital comments.

\subsection{D.Learn}

Otherwise, D.Learn purpose is to engage students ready to undertake a higher-level education in design and, therefore, who are more interested in the didactic aspects of the discipline, such as its complexity, branches, interrelations and modalities of development, mostly characterized by teamwork. Therefore, a role-play competitive game better suits these necessities. D.Learn involves four teams (representing the different courses of Politecnico di Milano: product, interior, communication and fashion design) confronting each other and composed by three members. This kind of experience requires the players to identify in a character and to collaborate with others to complete the game, acting accordingly to both their role and their team's specialization as a metaphor of a designer's daily work. A participant can play as: the navigator, who is provided with a smartphone and has to correctly locate the PoIs, control the position of the other teams and generally interact with the device; the strategist with a physical map of Milan city centre, who needs to plan the team's activities taking in considerations the scores and locations of the questions; and the dealer, who is equipped with a card deck with useful information for the resolution of the game and its trials. Each team has to solve ten quizzes with different levels of complexity, which are divided into: seven related to the team's specialized path (product, interior, communication or fashion design) and three concerning other design practice roles. Differently from D.Hunt, D.Learn needs an organizational support to regulate the formation of the teams, to provide the physical game kit, and to assure that every game session begins simultaneously, lasting about one and a half hour.

The starting point is Piazza del Duomo, then the game spreads across the city centre, according to each team's planning, through always visible PoIs: museums, showrooms, hotels, graphics, urban indoor and outdoor with clear meanings in relation to the design topic. It is patent that this game is more focused on challenging than on narrative features, and the educational goal is developed through a series of trials and thoroughly stimulated 
by the competition among the teams. In order to prevail, it is necessary to reach the highest score. Points and skills can be obtained along the way by correctly answering questions (always with the aid of hints on the cards); by visiting rewarding places, where the players acquire information on important brands, products, interiors, designers, graphics, or simple curiosities; or by stealing points to the opponents. In fact, team clashes are possible inside the gaming area, and the team with a highest score steals points from the other. That is why the players are more and more encouraged to reach the largest number of locations and give the right answers, actually seriously learning in an informal context Finally, the game session ends at Piazza dei Mercanti, where a final riddle has to be solved: the decryption of a code, for which the cards and the paper map are fundamental. The evaluation of this experience from the research team has been far easier than the previous one. As it required from six to twelve players to organize one game session and it needed control to start at the same time, a more direct contact with the players was inevitable. The researchers supervised the teams to record the experience and to guarantee the safety of the players, since most of them were underage. In addition, post-game interviews were organized.

\section{Results}

As already mentioned, the two games have been investigated in different ways, hence leading to different kinds of results. If D.Hunt evaluation is based on about one hundred one to five stars and tweet-like feedbacks, which could be quantitatively relevant but not completely reliable for drawing inferences; D.Learn, instead, counts on less played sessions, valuable from a qualitative perspective as stemmed from informal interviews and direct observation. Thus, only the latter will be discussed.

Primarily, the principle aims of the research - related to informal learning and the promotion of Milan design-related matters among interested high-school students - have been assessed. In fact, unknown design notions attracted the attention and were reported of increasing the knowledge of the participants, both because the system was designed to accompany students in a structured process of learning and comprehension, giving them the opportunity to answer the questions correctly and, mostly, because of the active involvement triggered by the game mechanics and real time, physically experienced collaboration and competition. As direct observation proved, players were so motivated that they carried on with the game despite the playground was considerably wide. Additionally, they expressed appreciation on the contextuality of the game: information provided close to the place it referred to, thanks to location-based dynamics, allowed a more meaningful learning and an active exploration of the city, bringing players to notice details they had never seen before and to build new personal geographies, overlaying the actual city with game contents (Montola, Stenros, \& Waern, 2009). Another important feature of D.Learn is the integration of digital contents with physical tools, to be used together in order to 
succeed in the game. This represented a further incentive for involvement, putting the digital and physical realities in contact, and, also, for social engagement. As a matter of fact, the digital device alone nurtures a self-referential focus, while physicality fosters an extroverted attitude, encompassing contextuality and social sharing (Petrelli et al., 2013; Spallazzo \& Mariani, 2018). In this specific case, the physical facilitators and the designed role-play mechanics forced the players to exchange information, skills and resources, inducing a discussion on the topics and, therefore, the building of a shared understanding and learning process, also reinforcing the team spirit. Thus, despite the limitations in the evaluation of the two games, it is possible to state that the project achieved good results in respect to the defined aims, leading to the valorisation and contextualisation of Italian Design throughout Milan and highlighting how culture can be diffused in an informal and playful way.

\section{Discussion}

The illustrated project has opened didactic experimentations that have been discussed in other papers. Though, it still has not fully exploited its significance. In fact, it gathers aspects that contemporary technological implementations in education should reckon. First, modern education should get passed conventional modalities to respond to the necessities of a rapidly changing society heading to automation. In this sense, informal game-based learning represents a valid alternative to a data-based education. It involves engaging experiences and narratives that encourage learners' motivation; if properly employed, exploiting different sources of information - physical spaces and tools, digital contents and the player's mind (De Souza e Silva, 2006; De Souza E Silva \& Sheller, 2015) -, it can enhance sociality, through collaboration or competition; and thanks to mobile technologies, situatedness and contextuality can be added values. By the way, technological devices alone risk to be exclusively self-referenced, isolating the users in a digital dimension, while the integration of real-life components (social or environmental) always has to be taken in consideration for actual engagement and learning.

In conclusion, the presented projects are intended to stimulate further reflections on future applications of spreading technologies in education, taking advantage of past experience.

\section{References}

Anastasiadis, T., Lampropoulo, G., \& Siaka, K. (2018). Digital Game-based Learning and Serious Games in Education. International Journal of Advances in Scientific Research and Engineering, 4(12), 139-144.

De Souza e Silva, A. (2006). Re-Conceptualizing the Mobile Phone - From Telephone to $\begin{array}{llll}\text { Collective } \quad \text { Interfaces. } & \text { Society, }\end{array}$ https://doi.org/10.1177/009430610603500209 
De Souza E Silva, A., \& Sheller, M. (2015). Introduction. Moving towards adjacent possibles. In A. De Souza E Silva \& M. Sheller (Eds.), Mobility and Locative Media: Mobile Communication in Hybrid Spaces (1 edition, pp. 1-15). Routledge.

Dickens, C. (1854). Hard Times. London: Bradbury \& Evans.

Gee, J. P. (2003). What Video Games Have to Teach Us about Learning and Literacy. Basingstoke: Palgrave Macmillan.

Gee, J. P. (2004). Situated Language and Learning: A Critique of Traditional Schooling (1 edition). New York: Routledge.

Huizenga, J., Admiraal, W., Akkerman, S., \& Dam, G. T. (2009). Mobile game-based learning in secondary education: engagement, motivation and learning in a mobile city game. Journal of Computer Assisted Learning, 332-344.

Klopfer, E. (2008). Augmented learning: research and design of mobile educational games. Cambridge, MA,: MIT Press.

Klopfer, E., Squire, K., \& Jenkins, H. (2002). Environmental Detectives: PDAs as a window into a virtual simulated world. In IEEE International Workshop on Wireless and Mobile Technologies in Education Proceedings (pp. 95-98).

Lave, J., \& Wenger, E. (1991). Situated learning: Legitimate peripheral participation. Cambridge: Cambridge University Press.

Mariani, I. (2014). Gioco e società. In M. Bertolo \& I. Mariani, Game Design. Gioco e giocare tra teoria e progetto. Milano: Pearson.

McGonigal, J. (2011). Reality Is Broken: Why Games Make Us Better and How They Can Change the World. New York: The Penguin Press.

Montola, M., Stenros, J., \& Waern, A. (2009). Pervasive Games. Expreriences on the Boundary Between Life and Play. Burlington,: Morgan Kaufmann Publishers.

Nafea, I. T. (2018). Machine Learning in Educational Technology. In H. Farhadi (Ed.), Machine Learning - Advanced Techniques and Emerging Applications (pp. 175-183). IntechOpen.

Petrelli, D., Dulake, N., Marshall, M., Willox, M., Caparrelli, F., \& Goldberg, R. (2013). Prototyping tangibles: exploring form and interaction (pp. 41-48). ACM Press. https://doi.org/10.1145/2540930.2540966

Prensky, M. (2001). Digital game-based learning. New York: McGraw-Hill.

Read, J. C. (2015). Serious games in education. EAI Endorsed Transactions on Serious Games, 2(6).

Spallazzo, D., \& Mariani, I. (2018). Location-Based Mobile Games. Design Perspectives. Cham: Springer. https://doi.org/10.1007/978-3-319-75256-3

Tuomi, I. (2018). The Impact of Artificial Intelligence on Learning, Teaching, and Education (EUR - Scientific and Technical Research Reports No. JRC113226). Luxembourg: Publications Office of the European Union.

Ulicsak, M., \& Wright, M. (2010). Games in education: serious games. Bristol: Nesta Futurelab. 